\title{
PARALLACTIC MOTION FOR COMPANION DISCOVERY: AN M-DWARF ORBITING ALCOR
}

\author{
Neil Zimmerman ${ }^{1,2}$, Ben R. Oppenheimer $^{1,2}$, Sasha Hinkley $^{3,11}$, Douglas Brenner ${ }^{2}$, Ian R. Parry ${ }^{4}$, \\ Anand Sivaramakrishnan ${ }^{2,5,6}$, Lynne Hillennbrand ${ }^{3}$, Charles Beichman ${ }^{7,8}$, Justin R. Crepp $^{3}$, Gautam Vasisht $^{8}$, \\ Lewis C. Roberts Jr. ${ }^{8}$, Rick Burruss ${ }^{8}$, David L. King ${ }^{4}$, Rémi Soummer ${ }^{9}$, Richard Dekany ${ }^{10}$, Michael ShaO ${ }^{8}$, \\ Antonin Bouchez ${ }^{10}$, Jennifer E. Roberts ${ }^{8}$, And Stephanie Hunt ${ }^{4}$ \\ ${ }^{1}$ Department of Astronomy, Columbia University, $550 \mathrm{~W} 120^{\text {th }}$ St, New York, NY 10027, USA; neil@ astro.columbia.edu \\ ${ }^{2}$ Astrophysics Department, American Museum of Natural History, Central Park West at W 79 ${ }^{\text {th }}$ St, New York, NY 10024, USA; bro@ amnh.org \\ ${ }^{3}$ Department of Astronomy, California Institute of Technology, 1200 E. California Blvd., MC 249-17, Pasadena, CA 91125, USA \\ ${ }^{4}$ Institute of Astronomy, University of Cambridge, Madingley Road, Cambridge CB3 OHA, UK \\ ${ }^{5}$ Department of Physics and Astronomy, Stony Brook University, Stony Brook, NY 11794, USA \\ ${ }^{6}$ NSF Center for Adaptive Optics, Santa Cruz, CA 95064, USA \\ ${ }^{7}$ NASA Exoplanet Science Institute, California Institute of Technology, Pasadena, CA 91125, USA \\ 8 Jet Propulsion Laboratory, California Institute of Technology, 4800 Oak Grove Dr., Pasadena CA 91109, USA \\ ${ }^{9}$ Space Telescope Science Institute, 3700 San Martin Dr, Baltimore, MD 21218, USA \\ ${ }^{10}$ Caltech Optical Observatories, California Institute of Technology, Pasadena, CA 91125, USA \\ Received 2009 November 11; accepted 2009 December 10; published 2010 January 6
}

\begin{abstract}
The A5V star Alcor has an M3-M4 dwarf companion, as evidenced by a novel astrometric technique. Imaging spectroscopy combined with adaptive optics coronagraphy allowed for the detection and spectrophotometric characterization of the point source at a contrast of $\sim 6 J$ - and $H$-band magnitudes and separation of $1^{\prime \prime}$ from the primary star. The use of an astrometric pupil plane grid allowed us to determine the projected separations between the companion and the coronagraphically occulted primary star to $\leqslant 3$ mas precision at two observation epochs. Our measurements demonstrate common parallactic and proper motion over the course of 103 days, significantly shorter than the period of time needed for most companion confirmations through proper motion measurements alone. This common parallax method is potentially more rigorous than common proper motion, ensuring that the neighboring bodies lie at the same distance, rather than relying on the statistical improbability that two objects in close proximity to each other on the sky move in the same direction. The discovery of a low-mass $\left(\sim 0.25 M_{\odot}\right)$ companion around a bright $(V=4.0 \mathrm{mag})$, nearby $(d=25 \mathrm{pc})$ star highlights a region of binary star parameter space that to date has not been fully probed.
\end{abstract}

Key words: binaries: general - instrumentation: miscellaneous - stars: individual (Alcor) - stars: low-mass, brown dwarfs - techniques: miscellaneous

\section{INTRODUCTION}

High-contrast imaging is a technique being developed for the study of faint objects in the vicinity of the closest stars to the Sun, to advance our understanding of binary stars, substellar companions, exoplanets, and circumstellar disks. For a recent discussion of this subject, see Oppenheimer \& Hinkley (2009). In general, the detection of a point source next to a bright star is insufficient evidence to establish a physical association. Over the years, a number of claims of companion detection relying only on single epoch observations and a measurement of color have subsequently been disproved through astrometric measurements. For example, the companion reported in the McCarthy et al. (1985) study of VB 8 was subsequently shown to actually be a background star (Perrier \& Mariotti 1987). As a result, researchers in this area have been careful to confirm through astrometry that any putative companion found shares the proper motion of the primary star, with orbital motion generally measured after several years of observations.

In fact, most of the stars in surveys for faint companions exhibit appreciable parallactic motion in addition to their proper motion. For example, over the course of one year, a star at a distance of $100 \mathrm{pc}$ will appear to trace an ellipse in the sky with a circumference of roughly 60 mas. The segment of the curve traversed by this star over an observation baseline of $\sim 3$ months

\footnotetext{
${ }^{11}$ Sagan Fellow.
}

provides an opportunity to discriminate against background stars in the same manner enabled by common proper motion analysis over longer timescales (e.g., Mugrauer \& Neuhäuser 2005). If the supposed companion maintains the same offset from the primary star over the duration of time between the observation epochs - to within an appropriate tolerance set by the upper limit of hypothetical orbital motion - then a strong argument can be made for the physical association of the two objects.

We note that the use of parallactic motion discrimination requires higher precision astrometry than has typically been possible in high-contrast imaging. For example, Thalmann et al. (2009) achieved a 10 mas level of precision and managed a detection of common parallax. Here we achieve a factor of 3 better precision to confirm an object's physical association. Other coronagraphs have not yet demonstrated similar levels of relative astrometry, with tens to hundreds of milliarcsecond astrometry being typical. This is particularly true when no other stars with well-established astrometric parameters lie in the field of view-a common situation that most high-contrast imaging devices face in the future (we note that with the aid of a fixed background star, Neuhäuser et al. (2008) measured a common parallax for GQ Lupi and its companion).

We have used the common parallax method to discover and confirm a companion orbiting the star Alcor (also known as HD 116842 and HIP 65477; J2000 coordinates $\alpha=$ $13^{\mathrm{h}} 25^{\mathrm{m}} 13.538, \delta=+54^{\circ} 59^{\prime} 16^{\prime \prime} \cdot 65$ in Perryman et al. 1997). 
Table 1

Summary of Project 1640 Observations of the Alcor System

\begin{tabular}{lccccc}
\hline \hline Mean UT Date & Besselian Year & $\tau_{\exp }(\mathrm{s})$ & $\lambda(\mu \mathrm{m})$ & $\rho$ (mas) & P. A. (Degrees East of North) \\
\hline 2009 Mar 16 10:35 & 2009.20469 & 1912 & $1.10-1.76$ & $1050 \pm 1$ & $206.5 \pm 0.1$ \\
2009 Jun 27 3:51 & 2009.48593 & 293 & $1.10-1.76$ & $1043 \pm 1$ & $207.1 \pm 0.1$ \\
\hline
\end{tabular}

Independently, Mamajek et al. (2010) imaged this same companion in the infrared $\mathrm{M}$ band, though their single epoch of data did not permit them to confirm the physical association with Alcor through astrometry. See the Appendix for a discussion of Alcor's rich role in the early stage of modern astronomy. While our astrometry measurements alone permit concrete affirmation of companionship, we also obtained low-resolution spectra and photometry in the $J$ and $H$ bands, completing the portrait and identifying the companion as an M3-M4 main-sequence star of roughly $0.25 M_{\odot}$. Although Alcor has been surveyed for possible companions in the past with speckle interferometry, the dynamic range of this technique at angular separations beyond several times the instrument's Rayleigh resolution limit is inferior to that obtainable with adaptive optics (AO) coronagraphy, as used in this study. For example, when McAlister et al. (1993) conducted speckle interferometry observations of Alcor with the $3.6 \mathrm{~m}$ CanadaFrance-Hawaii Telescope, their dynamic range was limited to 3 mag at separations $>00^{\prime} .04$, and consequently could not have detected the object we describe in this article. On the other hand, Lyot coronagraphs coupled with AO systems can routinely attain dynamic ranges of $\sim 10$ mag at a separation of $1^{\prime \prime}$ from the target star (Oppenheimer \& Hinkley 2009). Although few low-mass stellar companions to A stars such as Alcor have been imaged, with the increasing prevalence of high-contrast imaging surveys, recently other systems of similar nature have been found (e.g., S. Hinkley et al. 2010, in preparation; Kouwenhoven et al. 2005).

Alcor is a member of the nucleus of the Ursa Major (UMa) moving group. With a spectral type of $\mathrm{A} 5 \mathrm{~V}$, it is one of the seven main-sequence A stars with high confidence association to the group, based on kinematic and spectroscopic indicators (King et al. 2003). Despite the long history of studies of the UMa group, there remains a considerable uncertainty in the age of these stars. After compiling the photometry of a kinematically selected sample and comparing the resulting color-magnitude diagram with stellar evolution models, King et al. (2003) arrive at an age estimate of $500 \pm 100 \mathrm{Myr}$ for the group. Another recent study found that the color-magnitude diagram of the UMa group was best fit with an isochrone corresponding to an age of 400 Myr (Castellani et al. 2002). It should be noted that both of these age estimates are greater than the $300 \mathrm{Myr}$ ages obtained from earlier work (e.g., Soderblom \& Mayor 1993).

For several reasons, Alcor is an attractive target for highcontrast imaging surveys. First, the combination of close distance from the Sun, $24.9 \pm 0.4 \mathrm{pc}$ (Perryman et al. 1997), and its relatively young age (as mentioned above) increases the probability of detecting a previously unknown substellar companion: stars closer to the Sun have companions with larger angular separations on average, and, because substellar objects cool as they age, younger objects are easier to detect. (See, for example, the cooling characteristics in Burrows et al. 1998.) Furthermore, theoretical models of fragmentation in circumstellar disks suggest an abundance of low-mass companions around A stars (Kratter et al. 2009). Indeed, recent direct imaging discoveries of substellar companions support this hypothesis (Marois et al. 2008; Kalas et al. 2008).
The high apparent brightness of Alcor ( $V=4.0 \mathrm{mag}$ ) relative to other nearby stars is yet another agreeable feature. Highcontrast imaging surveys rely on the wave front correction provided by AO systems to attain large dynamic ranges within close angular separation of the target star. When the AO system uses on-axis light rather than an artificial guide star to measure the wave front errors caused by the atmosphere-as is the case of our study - the quality of the correction depends strongly on the brightness of the target star (Troy et al. 2000). For the above reasons, we chose to include Alcor in the Project 1640 survey of nearby stars.

\section{OBSERVATIONS}

Project 1640 is a near-infrared, Integral Field Spectrograph (IFS) situated behind an Apodized Pupil Lyot Coronagraph (APLC; Hinkley et al. 2008). During operation, Project 1640 is mounted behind the PALAO AO system (Dekany et al. 1997) on the 200" Hale Telescope at Palomar. The APLC consists of a pupil plane apodizer, a hard-edge focal plane mask, and a Lyot stop. The prolate apodization function and other masks are optimized to deliver broadband quasi-achromatic starlight suppression (Soummer 2005; Soummer et al. 2009). The APLC also includes a fine guidance system and an atmospheric dispersion corrector. In addition to the apodizer, another novel feature present in the pupil plane of the APLC is an astrometric grid that serves to indicate precisely the position of the star when it is occulted by the 370 mas diameter focal plane mask.

The grid of thin opaque lines in the pupil plane produces a periodic linear array of faint images of the obscured star along the symmetry axes of the grid, with the star itself at the intersection of two linear arrays (Sivaramakrishnan \& Oppenheimer 2006; Marois et al. 2006). These satellite spots form an array of stellar point-spread functions (PSFs) with angular spacing $\lambda / d$ ( $d$ being the line spacing, as projected back to the entrance pupil, $\lambda$ the wavelength of the light forming the image), and brightness approximately $(t / d)^{2}$ relative to the central unocculted PSF (where $t$ is the line thickness). We arranged to have the closest four satellite PSFs miss the focal plane mask but still lie within the field of view, to provide stable astrometric fiducials visible in every coronagraphic image.

Upon exiting the coronagraph, the optical beam passes through an array of $200 \times 200$ lenslets in the spectrograph. A dispersing prism produces an individual spectrum corresponding to each lenslet on the $2048 \times 2048$ pixel infrared detector, with a spectral resolution of $\lambda / \Delta \lambda \sim 30$ between $1.10 \mu \mathrm{m}$ and $1.76 \mu \mathrm{m}$ ( $J$ and $H$ bands). The detector subtends a field of view approximately $4^{\prime \prime}$ in diameter (Hinkley et al. 2008).

Table 1 summarizes our observations of Alcor. On 2009 March 16 we obtained 1912 s of data with Alcor occulted by the coronagraph, at an air mass of 1.10, under seeing conditions near $1^{\prime \prime}$. The AO system corrected this seeing such that images at $1.65 \mu \mathrm{m}$ exhibited a Strehl ratio of roughly $50 \%$. The pupil plane grid used during this observation produced four astrometric spots in the image at a brightness of $\sim 8$ mag fainter than the target star. A point source was immediately noticeable $\sim 1^{\prime \prime}$ from Alcor in the raw data. We observed Alcor again with good 


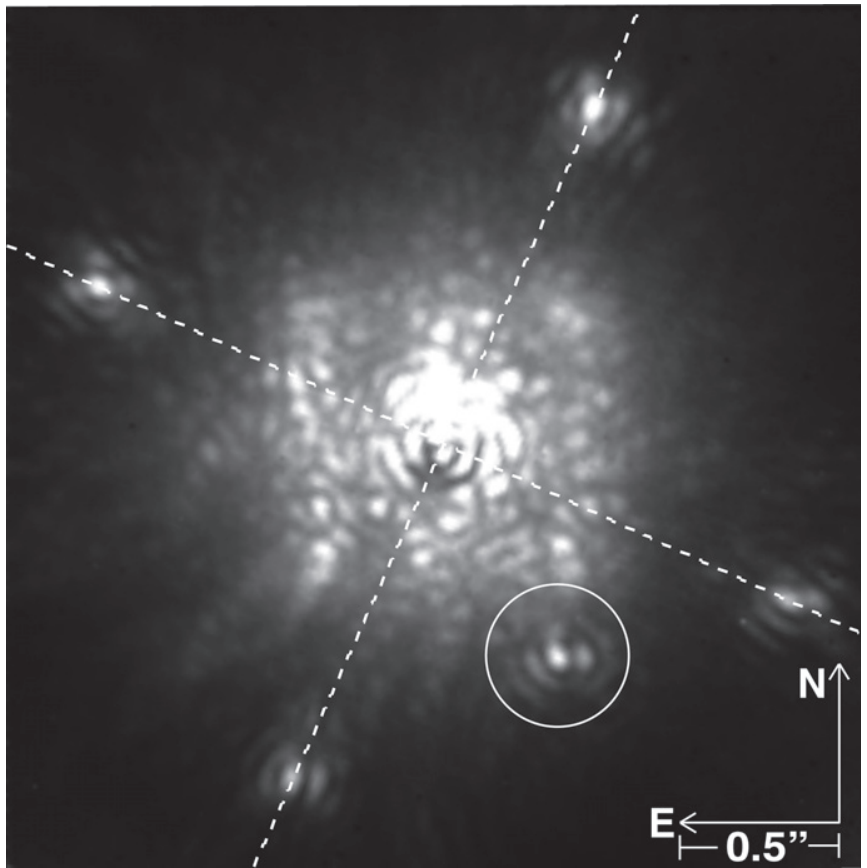

Figure 1. Coronagraphic image of Alcor obtained in June of 2009. This is the slice of the data cube corresponding to central wavelength $\lambda=1.61 \mu \mathrm{m}$. The dashed lines illustrate the intersection of the lines between the astrometric spots, indicating the position of Alcor behind the occulting mask. Coincidentally, the astrometric spots are approximately 6 mag fainter than the occulted star, similar to the brightness of the companion. The companion is the circled point source southwest of the image center.

atmospheric conditions on 2009 June 27, this time obtaining a total of 293 s of occulted data. Again the point source of interest was visible, in roughly the same location with respect to Alcor. During the June observations we used a pupil grid with thicker reticule wire, providing brighter astrometric spots, $\sim 6$ mag fainter than Alcor.

\section{DATA PROCESSING}

The Project 1640 IFS produces information with three dimensions simultaneously: two spatial and one spectral. Therefore, the most natural way to view the data is in the form of a cube where each slice is an image of the target field in a particular wavelength channel. We devised a data pipeline to automate the process of converting the detector images-each containing a mosaic of $4 \times 10^{4}$ closely packed spectra-into a data cube. The complete description of the details of this technique will be published elsewhere. Here we provide a general overview of how it works.

An essential component of the cube extraction is a library of images made by illuminating the IFS with a tunable laser. Each of these laser images contains the response of the IFS to a specific wavelength: a matrix of illuminated spots corresponding to the individual lenslets of the IFS. Effectively, they are keys showing what regions of the $4 \times 10^{4}$ spectra landing on the detector correspond to a particular central wavelength. The data pipeline uses the laser images to extract the science data and map them onto a cube, forming 23 images at wavelengths between 1100 and $1760 \mathrm{~nm}$, each with a bandwidth of $30 \mathrm{~nm}$. In addition to the mapping between the detector plane and the data cube, the Project 1640 data pipeline carries out numerous steps to prepare the data for analysis, including bias/dark-subtraction, bad pixel correction, and flat-fielding.
Figure 1 shows the $1.61 \mu \mathrm{m}$ slice of a data cube formed from our 2009 June 27 data. It is the result of 40 detector reads, each of duration $7.7 \mathrm{~s}$, for a total integration time of $293 \mathrm{~s}$. The four astrometric spots are visible on the peripheral of the image, while the point of source interest is detected southwest of the occulting mask. We aligned and co-added all the data from each epoch, producing one final data cube representing each epoch.

In the rest of the article we refer explicitly to "lenslet pixels" to describe the pixels comprising the data cube, to avoid ambiguity with the pixels on the detector of the IFS. Since each lenslet pixel constitutes a measurement of flux from an area element of the sky within a certain wavelength range, it can be treated in the same manner as the pixel of an ordinary digital image. Our analysis is done strictly on the data cubes that have already been extracted from the detector images, so the detector pixels are absent from further discussion.

\section{PHOTOMETRY}

In each spectral channel of the 2009 March 16 data cube, we performed aperture photometry on the putative companion. The residual light from the primary star significantly contaminated our image of the source. One component of this noise is in the form of speckles, which are not distributed in a smooth, easily modeled fashion (Racine et al. 1999; Perrin et al. 2003; Aime \& Soummer 2004; Hinkley et al. 2007; Soummer et al. 2007). Numerous efforts are underway to develop algorithms that remove speckles from IFS data by exploiting their chromatic properties (e.g., Sparks \& Ford 2002). However, these speckle suppression techniques have not yet matured enough to apply to data from an instrument such as ours without also altering the measured flux of true point sources. To minimize the effect of residual light from the primary star on our measurements, we counted the signal only in the core of the PSF, even though up to two Airy rings of the source diffraction pattern are apparent in the data (as in Figure 1). Since the PSF scales with wavelength, we used a different photometric aperture size for each half of the operating band to match the core size. For the first 11 channels (central wavelengths $1.10 \mu \mathrm{m}-1.40 \mu \mathrm{m}$ ), we measured the flux in a circle of radius 3 lenslet pixels, and used a 4 lenslet pixel radius circle for channels 12-23 (central wavelengths $1.43 \mu \mathrm{m}-$ $1.76 \mu \mathrm{m})$

In each channel of the data cube, the contaminating light from the primary star contributed $\sim 40 \%-50 \%$ of the flux counts within the core photometric aperture. To account for this, we subtracted a "background" estimate formed from the median of pixel values in the annulus between 16 and 19 lenslet pixels from the center of the source. The 16 lenslet pixel inner radius of this background annulus is outside the detected diffraction pattern of the point source of interest.

The uncertainty in the assumed level of contaminating light from Alcor based on the annulus median is the dominant source of error in the photometry. We estimated the uncertainty in the assumed contamination by measuring the scatter in the median values of carefully chosen patches of the channel images. These patches were at nearly the same lenslet pixel separation from Alcor as the putative companion PSF, contained within the 1619 pixel "background" annulus (so that they were beyond the influence of the putative companion PSF), and had the same area as our core photometric aperture. In other words, we based our uncertainty in the subtraction of the primary star's contribution by examining the behavior of its residual light in parts of the image that are subject to similar contamination to the core 
Table 2

Near-infrared Photometry of Alcor B

\begin{tabular}{lcc}
\hline \hline Band & $m$ & $M$ \\
\hline$J$ & $9.95 \pm 0.06$ & $7.97 \pm 0.06$ \\
$H$ & $9.56 \pm 0.06$ & $7.58 \pm 0.06$ \\
\hline
\end{tabular}

photometric aperture. We find the resulting error remains $\sim 5 \%$ of the companion signal across the band.

We derived $J$ - and $H$-band fluxes of the putative companion using a reference star observation to calibrate the photometry. On 2009 March 14, two days before our first epoch of Alcor data, we obtained a $7 \mathrm{~s}$ unocculted exposure of HD 107146/HIP 60074 (apparent magnitude $V=7.04$, spectral type G2V) at an air mass of 1.05 under similar observing conditions. Even though HD 107146 has a known debris disk, it is optically thin and only detected near our instrument's wavelengths in Hubble Space Telescope (HST) data (Ardila et al. 2004). The HST data show scattered light distributed in a ring of inner radius $3^{\prime \prime}$, which is outside our field of view and far beyond our $\sim 0{ }^{\prime} 1$ photometric aperture.

We carried out aperture photometry on the PSF in the HD 107146 data cube in an identical fashion as for the source in the Alcor image, using the same aperture and background annulus sizes. In the Two Micron All Sky Survey (2MASS) Point Source Catalog (Cutri et al. 2003), HD 107146 has $J$ - and $H$-band photometries listed as $5.87 \pm 0.02$ and $5.61 \pm 0.02$, respectively. We summed the core fluxes of the point source of interest and HD 107146 in the channel ranges corresponding to the 2MASS $J$ and $H$ filters $(1.13 \mu \mathrm{m}-1.34 \mu \mathrm{m}$ and $1.46 \mu \mathrm{m}-$ $1.73 \mu \mathrm{m}$, respectively). Subtracting the raw magnitudes of the HD $107146 J$ and $H$ sums from the 2MASS magnitudes, we derived correction magnitudes for each filter. Applying those corrections to the channel sums of the putative companion, we arrived at the broadband fluxes listed in Table 2 .

We estimated the probability of a star with matching photometric properties unassociated with Alcor coinciding with our field of view. One way to do this is to determine the surface density of point sources that have fluxes within the two-sided $5 \sigma$ confidence interval of our $J$ - and $H$-band magnitudes, corresponding to flux bounds $25 \%$ above and below our stated measurements. We queried the 2MASS Point Source Catalog for the number of $J$ - and $H$-band point sources in the $2^{\circ} \times 2^{\circ}$ area centered on Alcor's coordinates, separated into 1 mag wide bins extending between magnitudes 8 and 16. A linear regression fit to the logarithm of the source count as a function of magnitude yields the relations $\log _{10}\left(J\right.$-band sources deg $\left.{ }^{-2}\right)=-1.764+0.284 m_{J}$ with an rms residual of 0.167 , and $\log _{10}\left(H\right.$-band sources $\left.\operatorname{deg}^{-2}\right)=$ $-1.604+0.284 m_{H}$ with an rms residual of 0.090 . Integrating these point source surface density relations between the $5 \sigma$ flux boundaries of the supposed companion, 9.71 $\leqslant m_{J} \leqslant 10.26$ and $9.32 \leqslant m_{H} \leqslant 9.87$, we arrive at $J$-band and $H$-band point source surface densities $3.9 \mathrm{deg}^{-2}$ and $4.2 \mathrm{deg}^{2}$, respectively. Taking the larger of these two surface densities, $4.2 \mathrm{deg}^{-2}$, and multiplying by our $4^{\prime \prime} \times 4^{\prime \prime}$ field of view, we expect $5.2 \times 10^{-6}$ sources matching the photometric characteristics of the supposed companion in a given $4^{\prime \prime} \times 4^{\prime \prime}$ field of view in this part of the sky. Multiplying this by 100 to roughly account for the number of stars we have surveyed so far with null detections of stellar companions, we arrive at a posteriori probability of $0.05 \%$ that the source is unassociated with Alcor. Later in this article we will demonstrate how our astrometry reduces this probability to an even less significant quantity. With that knowledge in hand, we hereafter refer to the point source of interest as Alcor B, following the traditional nomenclature of directly imaged companions.

The parallax distance modulus of Alcor is $1.98^{\mathrm{m}}$, so to place Alcor B at the same distance implies it has absolute magnitudes $M_{J}=7.97 \pm 0.06$ and $M_{H}=7.58 \pm 0.06$. Henry \& McCarthy (1993) derived empirical mass-luminosity relationships for stars with masses between $0.18 M_{\odot}$ and $0.50 M_{\odot}$. When we apply these to our absolute $J$ - and $H$-band magnitudes, and take into account the variance inherent to the model and our own photometric uncertainty, we calculate mass estimates of $0.26 \pm_{0.07}^{0.10} M_{\odot}$ and $0.21 \pm_{0.03}^{0.04} M_{\odot}$, from the $J$ - and $H$-band luminosities, respectively. When we compare our fluxes to theoretical mass-luminosity models computed specifically for $600 \mathrm{Myr}$ old stars by Baraffe et al. (1998), similar to the published age estimates of Alcor, we find masses of $0.27 M_{\odot}$ and $0.25 M_{\odot}$, respectively. According to the mass-spectral class relationship for low-mass stars derived by Baraffe \& Chabrier (1996), a star with a mass between $0.2 M_{\odot}$ and $0.3 M_{\odot}$ indicates a spectral type in the range from $\mathrm{M} 2 \mathrm{~V}$ to $\mathrm{M} 3.5 \mathrm{~V}$.

\section{SPECTROSCOPY}

We extracted a low-resolution spectrum of the stellar companion from the IFS data. Again, we used the star HD 107146 as a reference source. As stated above, even though HD 107146 has a disk, it is faint and outside our field of view. Furthermore, the star lacks the excess emission that some disk hosts possess at $10 \mu \mathrm{m}$ (Metchev et al. 2004), so to the best of our knowledge, the spectrum is ordinary for a star of its class in our wavelength regime.

We began the spectral calibration by determining channelwise corrections for the wavelength-dependent transmission of the atmosphere and instrument. To do this, we made the assumption that at the spectral resolution of our data cube $(\lambda / \Delta \lambda \sim 30)$, and within our photometric errors, the spectrum of HD 107146 matches that of a typical G2V star. We compared our raw spectrum of HD 107146 with the measurement by Rayner et al. (2009) of the near-infrared spectrum of HD 76151, another G2V star. The HD 76161 data are part of a suite of reference stellar spectra collected at NASA's Infrared Telescope Facility (IRTF). We binned the publicly archived HD 76151 spectrum to our spectrograph's resolution, divided it into our raw HD 107146 spectrum, and mean normalized the result to obtain our response vector.

We obtained our raw spectrum of Alcor B by carrying out aperture photometry on the reduced data cube in the same manner as described in Section 4: counting the signal in an aperture containing the core of the PSF and subtracting the median of an annulus around the source multiplied by the aperture area. As before, our photometric errors were dominated by the uncertainty in the annulus estimate of Alcor's residual light in the companion photometric aperture, which includes a smooth halo and a speckle component.

We divided our raw Alcor B spectrum by the response vector to obtain the calibrated spectrum of the companion plotted in Figure 2. In our plot, we omit the five channels of our spectral range that are strongly subjected to variable telluric absorption, those with central wavelengths $1.10 \mu \mathrm{m}, 1.37-1.43 \mu \mathrm{m}$, and $1.76 \mu \mathrm{m}$. The spectrum data points are normalized to the mean of the included channels.

We compared the spectrum of Alcor B with a broad range of examples of M-dwarf spectra from the IRTF Spectral Library 


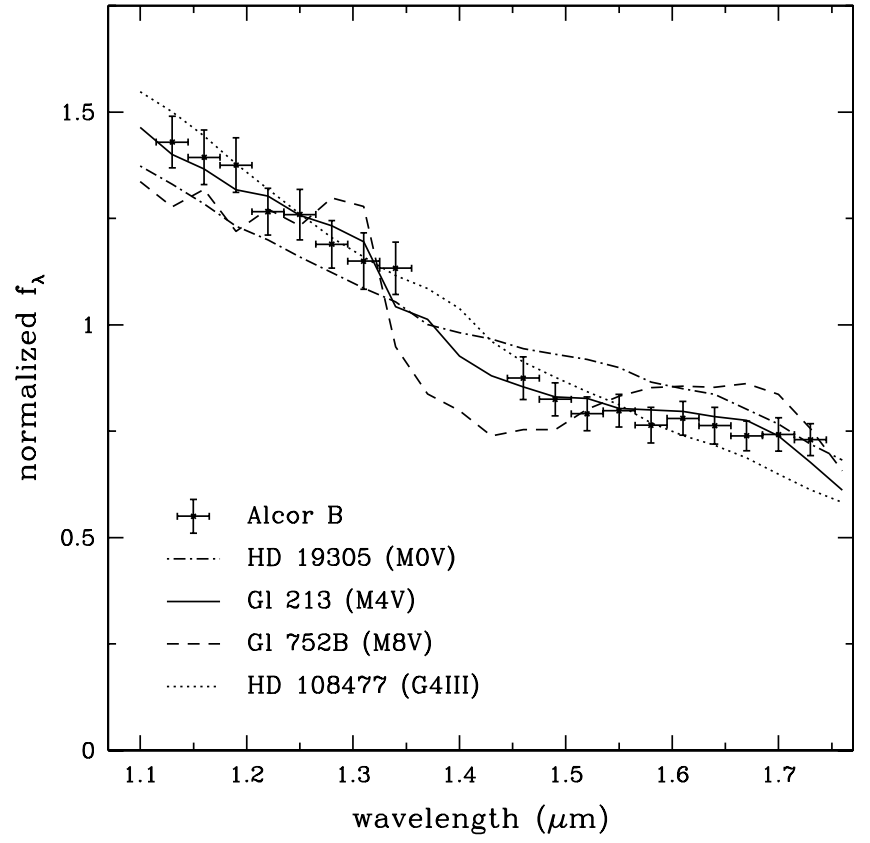

Figure 2. Spectrum of Alcor B extracted from the 2009 March data, compared with three examples of M-dwarf spectra and a giant spectrum whose $J-H$ color matches the photometry of the companion.

(Rayner et al. 2009). In addition, we compared our companion spectrum with that of the giant star in the IRTF Spectral Library with the closest $J-H$ color, HD 108477, a G4III star with a 2MASS $J-H$ color of 0.34 . After rebinning all of these comparison spectra to our data cube's spectral resolution, we normalized them and calculated the root mean square differences from the Alcor B spectrum. The spectra of three of these reference spectra are plotted alongside the Alcor B data in Figure 2. Of all of the spectra compared, the two closest matches to Alcor B are the M3.5V star Gl 273 and the M4V star Gl 213, both with root mean square residuals of $4 \%$. Although the shape of the G4III giant spectrum is qualitatively different from the Alcor B data, particularly in terms of the slope across $H$ band, its fit has an rms residual of only $5 \%$. This serves to indicate that at this spectral resolution there is ambiguity in discriminating between G-giant and M-dwarf stars of similar color.

\section{ASTROMETRY}

In each channel of the data cube, the intersection of the two perpendicular lines formed by the four astrometric grid spots determines the location of Alcor on the lenslet array (Figure 1). We compared these locations with the directly measured position of the companion in the data to measure the relative offset at each epoch. Due to a slight residual atmospheric dispersion causing an apparent drift in the position of the star by $\sim 2$ lenslets over the wavelength range in the data cube, we only compared the spot intersection with the position of the companion measured within the same channel. The companion and the grid spots have strongest detections in a subset of cube channels in the $H$ band, enabling the most accurate position determination at those wavelengths. These are also the wavelengths at which the atmospheric dispersion corrector is optimized. In addition, as is generally the case, the wave front correction of the $\mathrm{AO}$ system is better at $H$ band than in $J$ band. Therefore, we used only the five channels from $1.55 \mu \mathrm{m}$ to $1.67 \mu \mathrm{m}$-those spanning the $H$-band transmission peak-
Table 3

Relative Astrometry of Alcor B

\begin{tabular}{lccc}
\hline \hline \multicolumn{1}{c}{ Component } & 2009 March 16 & 2009 June 27 & Change \\
\hline East offset (mas) & $-470.3 \pm 3.1$ & $-476.3 \pm 2.9$ & $-6.0 \pm 4.3$ \\
North offset (mas) & $-939.1 \pm 1.7$ & $-928.2 \pm 1.5$ & $10.9 \pm 2.3$ \\
\hline
\end{tabular}

Note. The equatorial coordinate offsets of Alcor B relative to its host star on 2009 March 16 and 2009 June 27 (Besselian dates 2009.2047 and 2009.4859, respectively) followed by the change between the two epochs.

to deduce the relative offsets at each epoch. We measured the positions of the companion and the grid spots in the data cubes by fitting Gaussian profiles to the PSFs. For each epoch, we took the mean of the offsets between the grid spot intersection and the companion PSF in the five aforementioned channels to arrive at our final estimates. We then applied the Student's $t$ distribution (as appropriate when estimating a mean from a sample of five measurements-see Dean \& Dixon 1951) to derive $68 \%$ confidence intervals based on the standard deviation of the offset components between the channels.

To convert the lenslet pixel offsets into angular offsets oriented with equatorial coordinates, we applied our plate scale of $19.2 \pm 0.1 \mathrm{mas} /$ lenslet, and compensated for the rotation of our detector (the columns of the extracted data cubes are oriented $70.6 \pm 0.1$ counterclockwise with respect to north). Both the plate scale and rotation were derived from a series of observations of calibration binary systems with Grade 1 orbit solutions in the USNO Sixth Orbit Catalog (Hartkopf et al. 2001) between July 2008 and March 2009. Standard errors were propagated through all calculations to reflect $68 \%$ confidence intervals in the error bars. In Table 3, we list the resulting offset components between Alcor B and its host star.

As described in Section 4, if we consider only our photometric measurements of the putative companion, we are left with a small possibility $(\sim 0.05 \%)$ that it is an unassociated star coinciding with our line of sight. Now, with our astrometry, we can rule out this possibility to a stronger degree, in order to affirm the physical association with Alcor.

First, as illustrated in Figure 3, we can rule out the simple notion that the supposed companion is actually a distant background star lacking significant proper or parallactic motionone that is, for our purposes, fixed on the sky. For example, one could imagine a luminous star at a distance of $\sim 1 \mathrm{kpc}$, whose parallactic motion between our two observations is only $\sim 1.5$ mas, and whose projected space motion also happens to be near or below our astrometric precision. By contrast, over our 103 day baseline, the parallactic and proper components of Alcor's motion (see Table 4) resulted in a displacement with a magnitude of 34 mas. Because our two images remained centered on Alcor over the course of its motion, a fixed background star in our data would appear to shift about 34 mas relative to Alcor. More specifically, since the overall apparent motion of Alcor between our observations was 22.7 mas west and 25.2 mas south, a fixed background star lying southwest of Alcor would have shifted 22.7 mas east relative to Alcor (decreasing the magnitude of its offset from Alcor in right ascension), and 25.2 mas north relative to Alcor (decreasing the magnitude of its offset from Alcor in declination). The arc labeled $(\mu+\pi)_{\mathrm{BKG}}$ in Figure 3 represents this circumstance. Instead, we observed a westward motion of $6.0 \pm 4.3$ mas relative to Alcor and a relative northward motion of only $10.9 \pm 2.3$ mas (the two positions are labeled "March" and "June" in Figure 3). So the observed motion is inconsistent with a background star exhibiting 


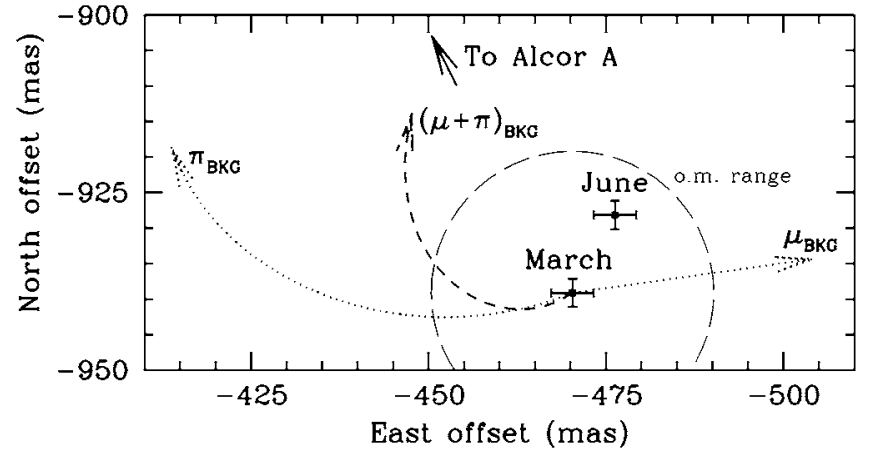

Figure 3. Summary of astrometry measurements, plotted in terms of north and east offsets from Alcor. The two position measurements of Alcor B are plotted with their associated $1 \sigma$ error bars, and labeled March and June, corresponding to UT epochs 2009 March 16 and 2009 June 29, respectively. The $\mu_{\mathrm{BKG}}$ and $\pi_{\mathrm{BKG}}$ arcs show the expected change in offset of a fixed background star due to Alcor's proper and parallactic motion. The $(\mu+\pi)_{\mathrm{BKG}}$ arc is the resultant of these components over the course of our two observation epochs. We have also plotted a circle labeled "o.m. range" containing an estimate of the upper limit of orbital motion with respect to the March position.

a low apparent motion on the order of several milliarcseconds or less.

Now we consider the case of a distant background star that does exhibit significant apparent motion, in a such a way that matches the observed displacement of the companion star between our observation epochs. The least luminous giant with consistent $J-H$ color, a star of type G2III, would have to be at a distance of about $740 \mathrm{pc}$ in order for its apparent magnitude to be consistent with our photometry. By combining our measurement of the change in Alcor B's offset from Alcor A and our knowledge of the apparent motion of the primary star, we can deduce the absolute motion of the putative companion on the sky, decoupled from Alcor: $28.6 \pm 4.3$ mas west and $-14.4 \pm 2.3$ mas south. At a distance of $740 \mathrm{pc}$, the expected parallactic motion between our observation epochs is 1.9 mas west and 0.7 mas south. Then, a proper motion of 26.7 mas west and 13.7 south is needed to make up for the difference from the observed apparent motion. We compute the space velocity from this assumed proper motion and distance using the formulae described in Johnson \& Soderblom (1987). Assuming zero radial velocity, this star would need a galactic space velocity of $U=-150 \mathrm{~km} \mathrm{~s}^{-1}, V=-300 \mathrm{~km} \mathrm{~s}^{-1}$, and $W=$ $130 \mathrm{~km} \mathrm{~s}^{-1}$ to be consistent with the apparent motion we measure. The largest component of this space velocity, $V$, indicates a strong retrograde galactic orbit. For more luminous giant stars, the necessary space velocities grow to even more unlikely values - a K1III giant, for example, would need a $V$ component of $-600 \mathrm{~km} \mathrm{~s}^{-1}$ to be consistent with our astrometry. In that case, $V$ is within the range of estimates of the local escape speed of the galaxy (e.g., $498 \mathrm{~km} \mathrm{~s}^{-1}<v_{\text {esc }}<$ $608 \mathrm{~km} \mathrm{~s}^{-1}$ from Smith et al. (2007) and $489 \mathrm{~km} \mathrm{~s}^{-1}<v_{\mathrm{esc}}<$ $730 \mathrm{~km} \mathrm{~s}^{-1}$ from Kochanek 1996).

The only plausible scenario remaining, that we have in fact discovered a low-mass companion to Alcor, can be checked by comparing the measured relative motion to Alcor with an estimate of the upper limit on the orbital motion a true companion would exhibit between our two observation epochs. The empirical mass-luminosity relation for intermediate-mass stars of Malkov (2007) implies a mass of $1.8 M_{\odot}$ for the A5V primary star, given its absolute magnitude $M_{V}=2.01$. Assuming a mass of $0.25 M_{\odot}$ for the companion, a circular orbit of the projected radius $26 \mathrm{AU}$ ( 1 .'05 at $24.9 \mathrm{pc}$ ) would have
Table 4

Apparent Motion of Alcor Between Observation Epochs

\begin{tabular}{lc}
\hline Component $^{\mathrm{a}}$ & ${\text { Change }(\mathrm{mas})^{\mathrm{b}}}^{\mathrm{b}}$ \\
\hline$\Delta$ East $_{\mathrm{PM}}$ & 33.9 \\
$\Delta$ North $_{\mathrm{PM}}$ & -4.8 \\
$\Delta$ East $_{\pi}$ & -56.5 \\
$\Delta$ North $_{\pi}$ & -20.4 \\
$\Delta$ East $_{\mathrm{PM}}+\pi$ & -22.7 \\
$\Delta$ North $_{\mathrm{PM}+\pi}$ & -25.2 \\
\hline
\end{tabular}

Notes.

a Subscripts PM and $\pi$ indicate the expected proper and parallactic motion, respectively. The subscript $\mathrm{PM}+\pi$ indicates the total displacement due to combined proper and parallactic motion.

b Based on values from the Hipparcos Catalog (Perryman et al. 1997), applied to epochs 2009 March 16 and 2009 June 27 (Besselian dates 2009.2047 and 2009.4859, respectively). The Hipparcos tables identify a proper motion of 120.35 mas $\mathrm{yr}^{-1}$ in R.A. (corrected for declination to reflect motion on a great circle), $-16.94{\text { mas } \mathrm{yr}^{-1}}^{-1}$ in decl. (with a $1 \sigma$ error of $<0.52$ mas $\mathrm{yr}^{-1}$ in each direction), and a parallax of $40.19 \pm 0.57$ mas.

a period of roughly 93 years, resulting in an apparent motion of $\sim 20$ mas if it were orbiting face-on. In fact, the motion we detected is smaller than this, but any inclination, eccentricity, or different semi-major axis in the orbit could change the expected orbital motion. However, most importantly, the apparent motion of Alcor B that we do detect is consistent with plausible orbital motion around Alcor. A circle illustrating the range of possible orbital motion with respect to the position of Alcor B at the first observation epoch is shown in Figure 3.

\section{DISCUSSION AND CONCLUSIONS}

Although we observed Alcor only twice over a baseline of 103 days, the high precision ( $\leqslant 3$ mas) relative astrometry enabled by the pupil plane grid of Project 1640 allowed us to find common parallactic and proper motion, thereby ruling out the possibility that the newly detected point source is a background star. We expect that as we improve our techniques for interpreting data from the IFS, we can attain yet higher astrometric precision in future studies. With sufficient sensitivity, such methods can be extended to lower mass objects, to characterize young, long period exoplanets. The rapidity of common parallax discrimination, as opposed to observation baselines $\gtrsim 1$ year relying on proper motion analysis alone, could improve the efficiency of future high-contrast imaging efforts. In particular, in the surveys that will be carried out with Project 1640 and the planned high-order AO system for Palomar, as well as the similar system planned for Gemini Observatory (Gemini Planet Imager; Macintosh et al. 2006), repeated observations of a faint companion candidates should be scheduled $\sim 1-4$ months from the initial detection epoch. This period is short enough for a typical target to remain visible in the night sky, but long enough to allow for sufficient parallactic motion for stars closer than $\sim 50 \mathrm{pc}$.

Under the most favorable observation arrangements, where investigators can acquire high precision relative astrometry of a possible companion three or more times within several months of the discovery date, the common parallax technique can demonstrate physical association with yet greater rigor than we have achieved here. If the primary star traces a parallactic arc of sufficient curvature over the observation baseline, three 
epochs of data indicating a persistent offset vector can no longer be accounted for geometrically by a background star. In such a case, the celestial coordinate trajectory of a true companion would be seen to deviate from regular linear motion to an extent that cannot be explained by a masquerading background star, even one with the most anomalous space velocity. To thereby show that the discovered neighbor follows the arc of the host star's parallactic ellipse would demonstrate companionship most conclusively.

We note that recently Thalmann et al. (2009) also used common parallax measurements to confirm the existence of a companion to the star GJ 758. However, Thalmann et al. do not discuss the significance of this method in their article. Presumably since the coronagraph they used lacks an astrometric grid, they were not able to attain as high a precision in the relative position of the companion as achieved in our study, reporting an uncertainty of 9.5 mas.

We acquired a low-resolution $(\lambda / \Delta \lambda \sim 30)$ spectrum of the companion with the Project 1640 IFS, enabling a preliminary spectral classification of M3V-M4V. We demonstrated that even with significant contamination of host starlight, a low spectral resolution IFS can be effective in constraining the spectral type of newly discovered companions. A comparison between our broadband $J$ - and $H$-band fluxes with two different massluminosity relationships yielded mass estimates ranging from 0.21 to $0.27 M_{\odot}$. Unlike lower mass stars (e.g., Metchev \& Hillenbrand 2009), few systematic surveys have been carried out with AO-equipped telescopes to characterize the frequency and mass ratio distribution of binary A stars, so it is difficult to place the significance of this discovery in the context of established binary star properties.

The object we found is relevant to the conundrum of $\mathrm{X}$-ray emission from A stars. Unlike lower mass (F-M) mainsequence stars and $\mathrm{O}$ and $\mathrm{B}$ stars, there is no consensus on a physical mechanism for X-ray emission from A stars. They lack the energetic winds of more massive stars, which explain the commonly seen X-ray activity of O and B stars. They also lack the convection-driven magnetic dynamos of lower mass mainsequence stars, which are widely held to be the source of their X-ray emission (Pallavicini 1989). Despite this, 10\%-15\% of A stars were detected as X-ray sources by the Röntgen Satellite (ROSAT; Schröder \& Schmitt 2007). In fact, Alcor is one of them, detected in the ROSAT All Sky Survey, with an X-ray luminosity of $L_{X}=2.8 \times 10^{28} \mathrm{erg} \mathrm{s}^{-1}$. It has long been proposed that unseen lower mass companions could account for the anomalous X-ray emission of many of these A stars (Schmitt et al. 1985). When Patience et al. (2001) surveyed A stars for stellar companions with the U.S. Air Force Advanced ElectroOptical System (AEOS), they found previously unknown companions to 8 of the 11 observed A stars with known X-ray source coincidence. Our finding lends further support to the hypothesis that hidden stellar companions explain the majority of perceived A star X-ray activity.

The authors wish to express our appreciation toward Jean Mueller, Kajsa Peffer, Karl Dunscombe, and the Mountain Crew at Palomar Observatory. We thank Adam Burgasser and Emily Rice for sharing comparison spectra to aid in our classification of the companion star. We are also indebted to our anonymous referee and the Scientific Editor of the Journal, Steven Kawaler, for expediting our review process. Project 1640 is funded by National Science Foundation grants AST-0520822, AST-0804417, and AST-0908484. A portion of the research in this paper was carried out at the Jet Propulsion Laboratory, California Institute of Technology, under a contract with the National Aeronautics and Space Administration (NASA) and was funded by Internal Research and Technology Development funds. In addition, part of this work was performed under a contract with the California Institute of Technology (Caltech) funded by NASA through the Sagan Fellowship Program. The members of the Project 1640 team are also grateful for support from the Cordelia Corporation, Hilary and Ethel Lipsitz, the Vincent Astor Fund, Judy Vale, Andrew Goodwin, and an anonymous donor.

Facilities: Hale (PALAO, Project 1640)

\section{APPENDIX}

\section{HISTORY OF EARLY PARALLAX MEASUREMENT ATTEMPTS WITH ALCOR}

In celestial lore, Alcor is best known for the place it shares in the sky with Mizar in the handle of the "Big Dipper" asterism. Alcor and Mizar were commonly used in ancient times as a test of visual acuity (Bohigian 2008). The fainter Alcor, at a separation of $12^{\prime}$, cannot be discerned unless one has good natural eyesight or corrective glasses. The pair is collectively designated $\zeta$ Ursa Majoris in Johann Bayer's 1603 Uranometria star catalog. Although Alcor and Mizar share physical association in the UMa moving group, it has yet to be shown conclusively whether or not they are gravitationally bound. However, Mizar itself was the first true multiple star system to be resolved with a telescope, by Benedetto Castelli, a colleague of Galileo Galilei (Fedele 1949).

Alcor and Mizar were also among the subjects of the first attempts to measure stellar parallax. Well before the invention of the telescope, stellar parallax was identified as the most conclusive way to demonstrate the Copernican assertion that the Earth orbits the Sun (Siebert 2005). In 1597, Johannes Kepler wrote a letter to Galileo encouraging him to attempt stellar parallax measurements, hoping he would succeed where Tycho Brahe had failed. Galileo recognized that the field of view containing Mizar, Alcor, and Sidus Ludoviciana (also known as HD 116798) was ideal for parallax measurements. The three stars form an approximate right triangle and they are at high declination, meaning that parallactic motion would trace an ellipse of low eccentricity. The triangle provides a position reference in two spatial directions. Galileo spent considerable effort trying to measure an actual parallactic motion (Galilei 1967) and distances to stars, but never succeeded. See Siebert's (2005) article for more detail. Although these early attempts at measuring parallax were beyond the measurement precision of the time, it is somehow poetic that a new result concerning Alcor some 400 years later relies on parallax.

\section{REFERENCES}

Aime, C., \& Soummer, R. 2004, ApJ, 612, L85

Ardila, D. R., et al. 2004, ApJ, 617, L147

Baraffe, I., \& Chabrier, G. 1996, ApJ, 461, L51

Baraffe, I., Chabrier, G., Allard, F., \& Hauschildt, P. H. 1998, A\&A, 337, 403 Bohigian, G. M. 1998, Surv. Ophthalmol., 53, 536

Burrows, A., et al. 1998, in ASP Conf. Ser. 154, Cool Stars, Stellar Systems, and the Sun, ed. R. A. Donahue \& J. A. Bookbinder (San Francisco, CA: ASP), 27

Castellani, V., Degl'Innocenti, S., Prada Moroni, P. G., \& Tordiglione, V. 2002, MNRAS, 334, 193

Cutri, R. M., et al. 2003, The IRSA 2MASS All-Sky Point Source Catalog, NASA/IPAC Infrared Science Archive (Pasadena, CA: CalTech), http://irsa. ipac.caltech.edu/applications/Gator/ 
Dean, R. B., \& Dixon, W. J. 1951, Anal. Chem., 23, 636

Dekany, R. G., Wallace, J. K., Brack, G., Oppenheimer, B. R., \& Palmer, D. 1997, Proc. SPIE, 3126, 269

Fedele, U. 1949, Coelum, 17, 65

Galilei, G. 1967, Dialogue Concerning the Two Chief World Systems, Ptolemaic \& Copernican (translated by Stillman Drake; Berkeley, CA: Univ. California Press)

Hartkopf, W. I., Mason, B. D., \& Worley, C. E. 2001, Sixth Catalog of Orbits of Visual Binary Stars (Washington, DC: USNO), http://www.ad.usno. navy.mil/wds/orb6/orb6.html

Henry, T. J., \& McCarthy, D. W., Jr. 1993, AJ, 106, 773

Hinkley, S., Oppenheimer, B. R., Brenner, D., Parry, I. R., Sivaramakrishnan, A., Soummer, R., \& King, D. 2008, Proc. SPIE, 7015, 701519

Hinkley, S., et al. 2007, ApJ, 654, 633

Johnson, D. R. H., \& Soderblom, D. R. 1987, AJ, 93, 864

Kalas, P., et al. 2008, Science, 322, 1345

King, J. R., Villarreal, A. R., Soderblom, D. R., Gulliver, A. F., \& Adelman, S. J. 2003, AJ, 125, 1980

Kochanek, C. S. 1996, ApJ, 457, 228

Kouwenhoven, M. B. N., Brown, A. G. A., Zinnecker, H., Kaper, L., \& Portegies Zwart, S. F. 2005, A\&A, 430, 137

Kratter, K. M., Murray-Clay, R. A., \& Youdin, A. N. 2009, arXiv:0909.2644

Macintosh, B., et al. 2006, Proc. SPIE, 6272, 62720L

Malkov, O. Y. 2007, MNRAS, 382, 1073

Mamajek, E., et al. 2010, AJ, in press

Marois, C., Lafrenière, D., Macintosh, B., \& Doyon, R. 2006, ApJ, 647, 612

Marois, C., Macintosh, B., Barman, T., Zuckerman, B., Song, I., Patience, J., Lafrenière, D., \& Doyon, R. 2008, Science, 322, 1348

McAlister, H. A., Mason, B. D., Hartkopf, W. I., \& Shara, M. M. 1993, AJ, 106, 1639
McCarthy, D. W., Jr., Probst, R. G., \& Low, F. J. 1985, ApJ, 290, L9

Metchev, S. A., \& Hillenbrand, L. A. 2009, ApJS, 181, 62

Metchev, S. A., Hillenbrand, L. A., \& Meyer, M. R. 2004, ApJ, 600 , 435

Mugrauer, M., \& Neuhäuser, R. 2005, Astron. Nachr., 326, 701

Neuhäuser, R., Mugrauer, M., Seifahrt, A., Schmidt, T. O. B., \& Vogt, N. 2008, AAP, 484, 281

Oppenheimer, B. R., \& Hinkley, S. 2009, ARA\&A, 47, 253

Pallavicini, R. 1989, A\&AR, 1, 177

Patience, J., Macintosh, B. A., \& Max, C. E. 2001, Proc. SPIE, 4490, 178

Perrier, C., \& Mariotti, J.-M. 1987, ApJ, 312, L27

Perrin, M. D., Sivaramakrishnan, A., Makidon, R. B., Oppenheimer, B. R., \& Graham, J. R. 2003, ApJ, 596, 702

Perryman, M. A. C., et al. 1997, A\&A, 323, L49

Racine, R., Walker, G. A. H., Nadeau, D., Doyon, R., \& Marois, C. 1999, PASP, 111,587

Rayner, J. T., Cushing, M. C., \& Vacca, W. D. 2009, ApJS, 185, 289

Schmitt, J. H. M. M., Golub, L., Harnden, F. R., Jr., Maxson, C. W., Rosner, R., \& Vaiana, G. S. 1985, ApJ, 290, 307

Schröder, C., \& Schmitt, J. H. M. M. 2007, A\&A, 475, 677

Siebert, H. 2005, J. History Astron., 36, 251

Sivaramakrishnan, A., \& Oppenheimer, B. R. 2006, ApJ, 647, 620

Smith, M. C., et al. 2007, MNRAS, 379, 755

Soderblom, D. R., \& Mayor, M. 1993, AJ, 105, 226

Soummer, R. 2005, ApJ, 618, L161

Soummer, R., Ferrari, A., Aime, C., \& Jolissaint, L. 2007, ApJ, 669, 642

Soummer, R., et al. 2009, Proc. SPIE, 7440, 74400R

Sparks, W. B., \& Ford, H. C. 2002, ApJ, 578, 543

Thalmann, C., et al. 2009, ApJ, 707, L123

Troy, M., et al. 2000, Proc. SPIE, 4007, 31 